\title{
DIELECTRIC SPECTROSCOPY IN CRUSTAL ROCKS: PRELIMINARY RESULTS FROM NORTHEASTERN SICILY (ITALY) AND THE GULF OF CORINTH (GREECE)
}

\author{
Triantis D. ${ }^{1}$, Stavrakas I. ${ }^{1}$, Anastasiadis C. ${ }^{1}$, Vallianatos F. ${ }^{2}$, and Kershaw S. ${ }^{3}$ \\ 1 Department of Electronics Engineering. Technological Educational Institution of Athens, \\ Athens, 122 10, Greece, triantis@ee.teiath.gr \\ ${ }^{2}$ Department of Natural Resources \& Environment, Technological Educational Institution of \\ Crete, Chania, 73133, Greece, fvallian@chania.teicrete.gr \\ ${ }^{3}$ Department of Geography and Earth Sciences, Brunel University, Uxbridge, Midllesex, UB8, \\ 3PH UK, Stephen.Kershaw@brunel.ac.uk
}

\begin{abstract}
Dielectric response was studied on two samples of manganese- and iron-bearing rock materials of two different geological origins, one from Northeastern Sicily (Italy) and one from the Southern Gulf of Corinth (Greece). The study of the dielectric properties is a flexible non-destructive testing method. The technique is based on the application of a single- frequency $A C$ electric field to the sample and the measurement of the amplitude and phase of its response. In the present work the broadband dielectric relaxation spectroscopy technique was applied in the frequency range from $1 \mathrm{kHz}$ to $1 \mathrm{MHz}$. The hydrated samples, of the above rock materials, that give rise to high values of both the real and imaginary part of the complex dielectric permittivity function are due to interfacial polarization mechanisms attributed to pore water. The two dry samples exhibit much lower values with respect to the hydrated ones while they have a big differentiation between their own permittivity values as well as between the shapes of their dielectric responses. This can be attributed to different origins of geotectonic deformation of the samples.
\end{abstract}

\section{INTRODUCTION}

The study of the electrical properties of rocks has been developed as a considerable tool for the study of deep earth and upper crust. Electric characterization of geological structures has become an irreplaceable method for overcoming various problems that rise from this area of study. Several theoretical and experimental studies have been conducted in the frame of dielectric study of typical rock samples (Howell \& Licastro 1961, Knight 1983, Ruffet et al 1991a,b, Glover et al 1994a,b, Chelidze \& Gueguen 1999a,b, Lesmes \& Morgan 2001).

Typical rocks are porous geomaterials. While in vacuum they are excellent insulators but brinesaturated rocks exhibit conductivity many orders of magnitude bigger. Explanation of results of measurement of electrical properties is a difficult problem because of the complexity of pore geometry and due to the interfacial interaction between the solid and the liquid.

Dielectric Spectroscopy is a flexible tool, applicable as a non-destructive testing method (Jonscher 1983, Macdonald 1987). In this technique, a small single-frequency ac field is applied to a sample, and the amplitude and phase of the resulting current are measured. The amplitude of the ac signal is chosen to be small enough to assume a linear response of the material.

Thus, dielectric properties are of great interest for a great variety of samples and not only for those that are can be used for electrical applications (electrical insulators). The application of the DS method on rock samples is a potential tool since the possible superposition of various types of conduction and polarization mechanisms, both in volume and on the solid-liquid interface, complicates dielectric spectra and calls for model experiments, where the influence of a single factor can be traced reliably. At the same time, water-bearing rocks demonstrate unusual dielectric spectra, 
enormous static polarization and anomalously large relaxation times. Interfacial polarization is met in materials containing various phases (i.e. inhomogeneous).

Dielectric dispersion in heterogeneous conducting materials is traditionally described by the Maxwell-Wagner theory. (Maxwell 1892, Wagner 1914). Electrical field application makes the possibly free charges of the material to move to the interfacial zones creating long dipoles in the bulk of the material (i.e. Maxwell - Wagner polarization). This type of polarization is a common phenomenon on rocks since they contain many free charges in their structure because of their water content.

The theoretical models for the complex dielectric response of hydrated rocks constructed with respect to interfacial polarization mechanisms depend on the complex dielectric properties of the material phases and the effective shapes of the phases; they are independent of the grain and pore size. Additionally, the proposed theoretical models are satisfactory at modelling the frequencyindependent properties of conductive porous media (Hanai 1960, Sen et al 1981), while they are inadequate to explain the large dielectric enhancements and conductivity dispersions that are observed in conductive porous media at low frequencies. Micro-geometry affects current flow increasing the net polarisation within a sample, it can substantially increase the predicated dielectric enhancement of the interfacial polarisation models (Lysne 1983, Hilfer, 1991). Recent models have been proposed to predict the geometrical features of a porous medium from the dielectric response. (Hilfer 1996, Hilfer et al 2000).

Frequency dispersion $\mathrm{AC}$-impedance techniques are one of the most efficient tools to study the evolution of fracture generation and closing under in situ conditions. To this meaning the aforementioned method could be an index of the geotectonic environment since fracturing processes play crucial role in the geodynamic evolution. We note that the sensitivity of the method allows the detection of newly formed rock fractures (Glover et al., 1996). A detailed analysis is given by Nover et al., 2000 and Siddiqi et al., (1997).

The purpose of the present work is to demonstrate the applicability of dielectric spectroscopy as a potential tool for crustal rock characterization and to indicate that the DS method could be possibly contribute to the analysis of geotectonic deformation in geomaterials.

\section{DIELECTRIC SPECTROSCOPY}

Dielectric Spectroscopy measurements provide the values of complex permittivity of materials by applying on them an alternating electric field of various frequencies. Complex permittivity $\varepsilon^{*}$ is defined as follows

$$
\varepsilon^{*}=\varepsilon^{\prime}-\mathrm{j} \varepsilon^{\prime \prime}=\varepsilon^{\prime}+\left(\sigma / \mathrm{j} \varepsilon_{\circ} \omega\right)
$$

where $\varepsilon^{\prime}$ and $\varepsilon^{\prime \prime}$ are the real and imaginary parts of the complex permitivity correspondingly, $\sigma$ is the ac conductivity, $\varepsilon_{o}$ is the vacuum permittivity, $\omega$ is the angular frequency ( $\left.\omega=2 \pi f\right)$ where $f$ stands for the frequency and $j=\sqrt{-1}$. The real part of $\varepsilon^{*}$ is often met as relative dielectric constant denoted as $\varepsilon\left(\varepsilon=\varepsilon^{\prime}\right)$. The dependence of the real part $\varepsilon^{\prime}$ of the complex permittivity on frequency exhibits dielectric relaxation (or dielectric dispersion) in which $\varepsilon^{\prime}$ decreases with increasing frequency. The basic parameters of dielectric relaxation are the $\varepsilon_{S}$ and $\varepsilon_{\infty}$ low and high frequency limits of relative permittivity, respectively, and the relaxation intensity $\varepsilon_{s}-\varepsilon_{\infty}$ (or the relaxation magnitude). The imaginary part of $\varepsilon^{\prime \prime}$ (or loss factor) $\varepsilon^{\prime \prime}$ can also be described with respect to frequency showing a peak in the characteristic frequency $f_{m}$. If the dc conductivity $\left(\sigma_{D C}\right)$ is not negligible then the loss factor $\varepsilon_{d}^{\prime \prime}$ that expresses the dielectric losses due to dipole relaxations is calculated (Asami, 2002) from the equation

$$
\varepsilon_{d}^{\prime \prime}=\left(\sigma-\sigma_{D C}\right) / \varepsilon_{o} \omega
$$

The ratio of the imaginary part of $\varepsilon^{*}$ to the real part $\varepsilon^{\prime \prime} / \varepsilon^{\prime}$ is defined as the loss angle tangent $\tan \delta$ or dielectric loss

$\tan \delta=\varepsilon^{\prime \prime} / \varepsilon^{\prime}$

The complex plot (Cole-Cole diagram) is also used for the analysis of dielectric relaxation. In the Cole-Cole diagram the loss factor $\varepsilon^{\prime \prime}$ is plotted against relative permittivity and traces a semicircle if the dielectric relaxation has a single relaxation time (Debye type relaxation). Still, the Cole-Cole diagrams often deviate from semicircle implying relaxation time distribution. The deviation is expressed by various empirical equations proposed by several authors (Cole \& Cole 1941, Davidson \& Cole 1951, Havriliak \& Negami 1966). The most conventional empirical analytical dielectric ex- 
pression that is often used to describe the generalized broadened asymmetric relaxation loss peak observed in many dielectric materials over a wide frequency range is the Havriliak - Negami (HN) equation. This empirical equation (HN) is a combination of Cole-Cole and Davidson-Cole equations and constitutes a classification tool for dielectric relaxation of various materials and has the form

$$
\varepsilon^{*}(\omega)-\varepsilon_{\infty}=\left(\varepsilon_{\mathrm{s}}-\varepsilon_{\infty}\right) /\left(1+\left(j \omega \tau_{0}\right)^{1-\alpha}\right)^{\beta}
$$

where $\tau_{o}$ is a characteristic relaxation time $\left(\tau_{o}=1 / 2 \pi f_{o}\right)$ and $\alpha$ and $\beta$ parameters with values ranging between $0 \leq \alpha<1,0<\beta \leq 1$ stating the depression of the semicircle (Debye type relaxation) of the Cole-Cole plot. The parameter $\alpha$ is a measure of the broadness of a symmetric dielectric relaxation curve and $\beta$ is the shape parameter of an asymmetric relaxation curve.

In a different approach (Jonscher 1999) it was suggested, on the basis of experimental evidence, that the dielectric response in the frequency range, far from or in the absence of any loss peaks, of any dielectric solid, agrees with the following fractional power law

$$
\varepsilon^{*} \propto(i \omega)^{n-1} \text { with } 0<n<1
$$

which is usually referred as the "universal law".

\section{SAMPLES AND EXPERIMENTAL PROCEDURE}

The samples used are manganese- and iron-bearing precipitates collected from Holocene reefs from coastal northeastern Sicily (Aci Trezza, site near Catania, encoded as AT) and from the Gulf of Corinth (Mavra Litharia site encoded as ML). They were extracted from remnants of extensive growths preserved as encrustations on cliffs composed of a variety of bedrock. Coralline algae dominate reefs while a subsiduary fauna of calcified worms and solitary corals which are also attached. Reef frameworks are filled with fine-grained calcareous sediment together with cement fringes and micritic cements as well as small amounts of late-stage fine sand and mud. Lithification was achieved by a mixture of cement fringes and micritic cements (Kershaw, 2000). The used samples were shaped as slabs $4 \mathrm{~mm}$ thick and area of $800 \mathrm{~mm}^{2}$ approximately.

Two principle cases must be distinguished in laboratory measurements for the studied samples. Initial measurements have been conducted on hydrated samples without previous heating to remove water molecules distributed in the bulk of the samples. For the second set of experiments the same samples were used after heating for 6 hours at $400 \mathrm{~K}$ in a furnace. Dielectric permittivity measurements, were conducted immediately $(2 \mathrm{~min})$ after removing the samples from the furnace.

In the $2 \mathrm{~min}$ time the samples were weighed and after acquiring room temperature (295K) were placed in the dielectric test fixture. Weight measurements before and after sample heating proved that the water content was of the order of $0.52 \%$ and $0.44 \%$ for AT and ML samples correspondingly. This differentiation indicates that the AT sample absorbs more water than the ML sample. In order to investigate whether the absorption of water is a bulk or surface effect weight measurements were conducted before and after heating samples of different volume and of the same total area. The results of the measurements showed approximately the same percentage of water content for the corresponding samples. Thus the water molecules seem to be distributed within the bulk of the materials

Finally, a third set of weight measurements followed by a set of dielectric permittivity measurements were conducted two days after the removal of the samples from the furnace and maintained in atmospheric environment. It is characteristic that the AT and ML samples gain nearly the same water content $(0.50 \%$ and $0.43 \%$ respectively).

Electrical data were measured using a computer controlled LCR meter (Agilent model 4284A), accompanied by the dielectric test fixture (Agilent model 16451B) and further supported by a computer for data recording, storage and analysis. The dielectric test fixture that was used to hold the specimen was protected in a cabin providing constant humidity $(70 \%)$ and temperature $(295 \mathrm{~K})$. For all the dielectric measurements, the amplitude of a sinusoidal ac voltage source was kept constant at $2 \mathrm{~V}$. The instrument directly provides the values of parallel capacitance $C_{p}$ and the dielectric loss angle tangent $\tan \delta$. Seven frequencies per decade were selected with a logarithmic scaling in the frequency range $1 \mathrm{kHz}$ to $1 \mathrm{MHz}$ to perform the measurements. The real $\varepsilon^{\prime}$ and imaginary $\varepsilon^{\prime \prime}$ parts of dielectric permittivity were calculated using the respective equations and

$$
\varepsilon^{\prime}=4 \mathrm{t} \cdot \mathrm{C}_{p} /\left(\varepsilon_{\mathrm{o}} \cdot \pi \cdot \mathrm{d}^{2}\right)
$$


$\varepsilon^{\prime \prime}=\varepsilon^{\prime} \cdot \tan \delta$

(8)

where $t$ is the sample thickness, $d$ is the diameter of the used test fixture electrodes, $\varepsilon_{o}=8,856 \cdot 10^{-14} \mathrm{~F} \cdot \mathrm{cm}$ is the permittivity of free space and $\mathrm{f}$ is the frequency of the applied ac field.

\section{RESULTS AND DISCUSSION}

The variation of the real $\varepsilon^{\prime}$ and imaginary $\varepsilon^{\prime \prime}$ part of the complex dielectric permittivity $\varepsilon^{*}$ for the samples AT and ML, was studied as a function of the frequency of the applied electric field. Figure 1 presents the dependence of the real part $\varepsilon^{\prime}$ of the dielectric permittivity $\varepsilon^{*}$ on the frequency $f$ for the samples AT (Fig. 1a) and ML (Fig. 1b). Full circles correspond to the values of the $\varepsilon^{\prime}$ of the samples without being heated and maintained in constant temperature and humidity conditions. Empty circles correspond to the values of $\varepsilon^{\prime}$ and correspond to data taken after heating the samples at $400 \mathrm{~K}$ for $5 \mathrm{~h}$ to expel the captured water. The measurements were conducted immediately after the fast cooling ( $2 \mathrm{~min}$ ) of the sample in order to reach $295 \mathrm{~K}$ to avoid water accumulation.
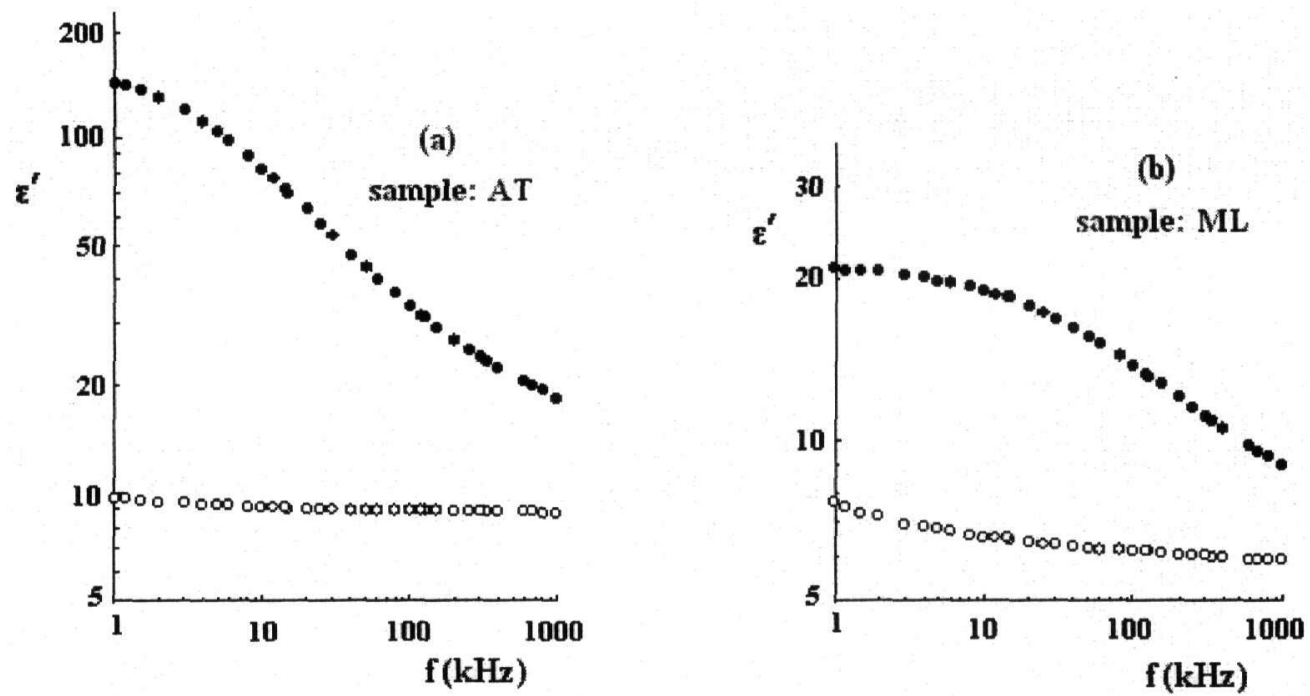

Figure 1. The real part $\varepsilon^{\prime}$ of the dielectric permittivity $\varepsilon^{\star}$ versus the frequency $f$ ((logarithmic plot) for the notheated samples (full circles[•]) and immediately after heating (empty circles[o]) .

When the samples are hydrated the striking feature of these plots is the increasing value of $\varepsilon^{\prime}$ especially in the range of frequencies between $10 \mathrm{kHz}$ and $1 \mathrm{MHz}$. For almost dry sample (after heating) the values of $\varepsilon^{\prime}$ in the same frequency range exhibit trivial dispersion. Slightly greater dispersion is observed for the ML sample which constitutes a first significant difference between the two kinds of studied samples. Another difference in the dielectric behavior of the dry samples is that are the values of $\varepsilon^{\prime}$ for the AT samples are greater than those of the ML samples. When the samples are left for 2 days in atmospheric humidity environment, an impressive change in the magnitude as well as in the shape of the plots $\varepsilon^{\prime}(f)$ is observed. The values of the real part of the complex dielectric permittivity at the corresponding frequencies return marginally to the values of the samples before heating. This significant change of the $\varepsilon^{\prime}(f)$ plots and the strong frequency dispersion exhibited by the hydrated samples can be attributed to surface currents along pore edges accompanied by interfacial charging at grain boundaries (Maxwell-Wagner effect).

Figure 2 depicts the dependence of the imaginary part $\varepsilon^{\prime \prime}$ of the dielectric permittivity $\varepsilon^{*}$ on the frequency $f$ for the AT (Fig. $2 a$ ) and the ML(Fig. $2 b$ ) samples. When the AT and the ML samples are hydrated (before heating or two days after heating them) reveal loss peaks at $6 \mathrm{kHz}$ and $80 \mathrm{kHz}$ correspondingly. 

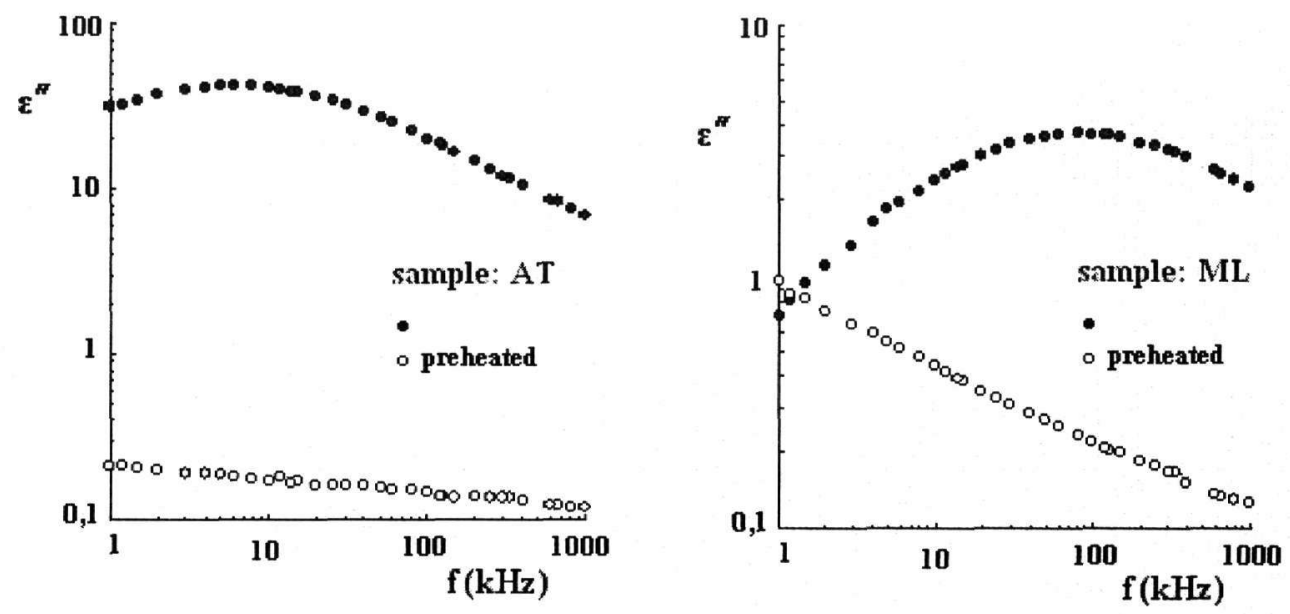

Figure 2. The imaginary part $\varepsilon^{\prime \prime}$ of the dielectric permittivity $\varepsilon^{*}$ versus frequency $f$ ((logarithmic plot) for the unheated samples (full circles[•]) and the heated samples (empty circles[o]).

Further study of the polarization mechanism of the hydrated AT and ML can be achieved by applying Havriliak - Negami (HN, Eq. 4) fitting to the experimental results. The results of this fitting for both samples are presented in the Cole-Cole diagrams of figure 3. Table 1 contains the values of the $\mathrm{HN}$ parameters for $\mathrm{AT}$ and $\mathrm{ML}$ samples extracted from the fitting process on the HN equation of the experimental data.
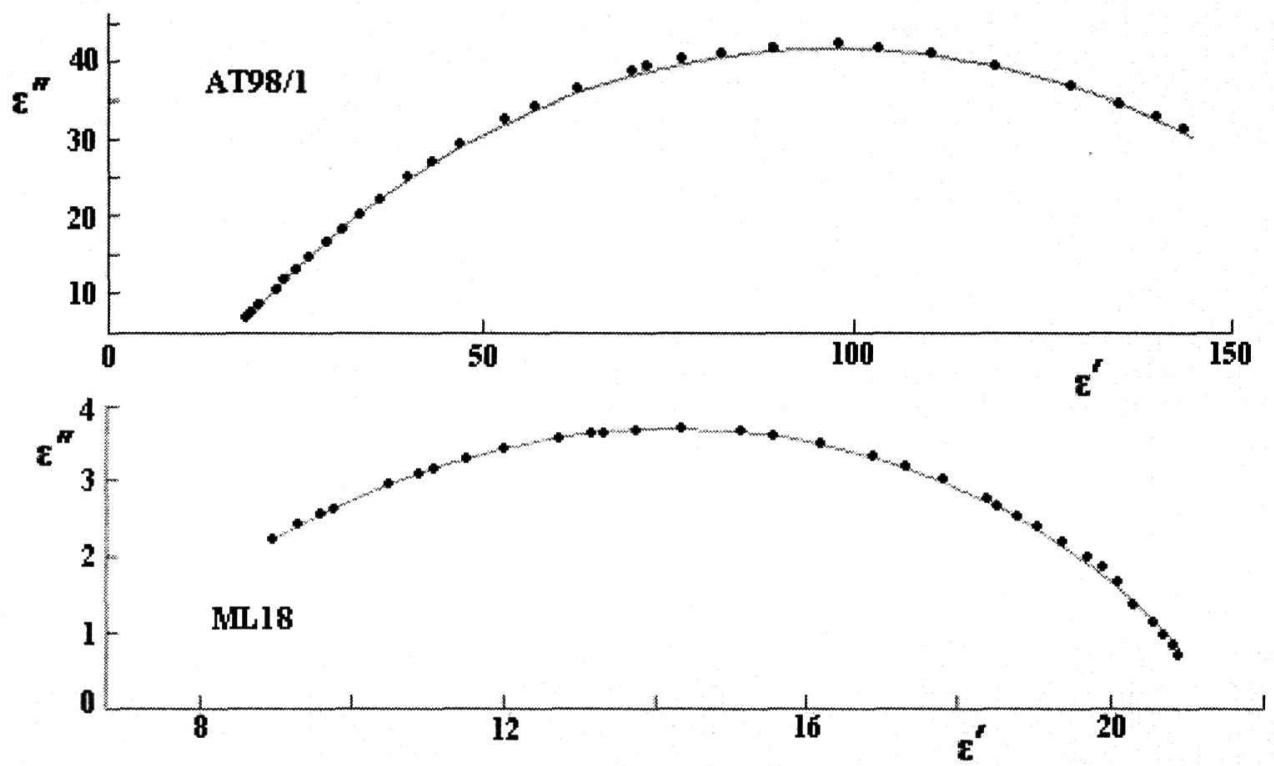

Figure 3. Dielectric response (Cole-Cole) diagrams of the hydrated samples AT and ML The circles [•] correspond to the experimental data and the solid lines are the curves fitted using the empirical equation HN [Eq. (4)].

It is impressing that the AT sample exhibits a greater relaxation magnitude $\varepsilon_{s}-\varepsilon_{\infty}$ than the ML sample, which means that the AT sample can be characterized by a higher degree of disorder 
in the polarization mechanism. This differentiation can be partially attributed to the larger water content of the AT than the ML sample. Another important difference is spotted on the parameters exponents $\alpha$ and $\beta$ of the HN empirical equation. For the AT sample since $\beta \approx 1$ a uniform distribution of the relaxation times can be observed with respect to the ML sample for which the shown distribution of relaxation times is depressed.

Table 1.: $\mathrm{HN}$ parameter values yield from the fitting procedure of the $\mathrm{HN}$ equation (Eq. 4) to the experimental data of the hydrated samples.

\begin{tabular}{cccccccc}
\hline Samples & $\varepsilon_{s}$ & $\varepsilon_{\infty}$ & $\varepsilon_{s}-\varepsilon_{\infty}$ & $f_{0}(\mathrm{kHz})$ & $\alpha$ & $\beta$ & $\mathrm{f}_{\mathrm{m}}(\mathrm{kHz})$ \\
\hline AT981 & 181 & 13.4 & 167.6 & 6 & 0.41 & 0.99 & 6 \\
ML18 & 21.5 & 6.0 & 14.7 & 58 & 0.39 & 0.78 & 80 \\
\hline
\end{tabular}

For the dry samples the values of $\varepsilon^{\prime \prime}(f)$ exhibit an increase as frequency decreases and far from any loss peaks in the range of $1 \mathrm{kHz}$ to $1 \mathrm{MHz}$. It appears to be a general rule that the prevailing forms of frequency dependence are fractional power laws (Eq. 5). Based on the fitting process of equation 5 to the experimental data, $\varepsilon^{\prime \prime}(f)$, the values calculated for the exponent $\mathrm{n}$ are 0.9 and 0.75 for the AT and ML samples respectively. The fact that the exponent $n$ varies in the range 0.71 means that the slowly mobile hopping charge carriers dominate polarization. The noticeable difference of the exponent $n$ values for the dry samples constitutes the main difference in the dielectric response of the two samples, given that in polarization and conduction mechanisms the absorbed water molecules are not dominant. The exponent $n$, could be used as a degree of geotectonic deformation applied to the sample. The degree of fragmentation in the samples from the Gulf of Corinth (samples ML) is higher than that of Sicily's (samples AT).

\section{CONCLUSIONS}

The dependence of the dielectric behaviour on hydration of the samples from Sicily (samples AT) and the Gulf of Corinth (samples ML) was studied at $295 \mathrm{~K}$ in a wide range of frequencies ( $1 \mathrm{kHz}$ to $1 \mathrm{MHz}$ ). The different dielectric response of the two samples illustrates that they exhibit different hydration levels at atmospheric conditions. The dry samples exhibit much lower values of the real and imaginary parts of the complex dielectric permittivity, than the hydrated ones. For the two dry samples, although the dielectric permittivity is described by the same power - law function of frequency, the difference on the value of the exponent $n$ is related to both the flatness of the dielectric loss in frequency and the degree of geotectonic deformation applied to the sample. The significance of these results is under investigation by the authors; we presume there are probably ocean chemistry variations that control the character of formation of the manganese and iron precipitates, although microbial control cannot be excluded, from the present small samples that have been analyzed here using the dielectric relaxation spectroscopy technique.

The interconnection of all the aforementioned parameters is a matter of further investigation in a ensemble of samples, covering the variety of geoenviromental conditions.

Acknowledgments: Authors would like to thank Mr. P. Kyriazis for his contribution in constructing the data fitting software.

\section{REFERENCES}

Asami, K., 2002. Characterization of heterogeneous systems by dielectric spectroscopy, Prog. Polym. Sci, 27, 1617-1659.

Chelidze, T. L., \& Gueguen, Y., 1999. Electrical spectroscopy of porous rocks: a review- I. Theoretical model, Geophys. J. Int., 137, 1-15.

Chelidze, T. L., Gueguen, Y., \& Ruffet, C., 1999. Electrical spectroscopy of porous rocks: a review- II. Experimental results and interpretation, Geophys. J. Int., 137, 16-34.

Cole, K.S., \& Cole, R.H., 1941. Dispersion and absorption in dielectrics, J. Chem. Phys., 9, 341-351.

Davidson, D.W., \& Cole, R.H., 1950. Dielectric relaxation in glycerine, J. Chem. Phys., 18, 1417pp.

Davidson, D.W., \& Cole, R.H., 1951. Dielectric relaxation in glycerol, propylene glycol and n-propanol, J. Chem. Phys., 19, 1484-1490. 
Glover, P.W.J., Meredith, P.G., Sammonds, P.R., \& Murrel, S.A.F., 1994a. Ionic surface electrical conductivity in sandstone, J. geophys. Res., 99 (B11), 21635-21650.

Glover, P.W.J., Meredith, P.G., Sammonds, P.R., \& Murrel, S.A.F., 1994b. Measurements of complex electrical conductivity and fluid permeabilities in porous rocks at raised confining pressures, in Rock Mechanics in Petroleum Engineering, Proc. EUROROCK94, Delft, Balkema, Amsterdam, pp. 29-36.

Glover, P.W.J., Gomezm J.B., Meredith, P.G., Boon, S.A., Saumonds, P.R. \& Marbell, S.A.F., 1996. Modeling the stress strain behaviour of saturated rocks undergoing triaxial deformation using complex electrical conductivity measurements, Sur. Geophys., 17 (3), 307-330.

Gueguen, Y., \& Palciauskas, V., 1994. Introduction to the Physics of rocks Princeton University Press, Princeton.

Havriliak, S., \& Negami, S., 1966. A complex plane analysis of $\alpha$-dispersions in some polymer systems, J. Polym. Sci., C, 14, 99-117.

Hilfer, R., 1996. Transport and Relaxation Phenomena in Porous Media, Adv. Chem.Phys, XCII, pp 299.

Hilfer, R., Widjajakussuma, J., \& Biswal, B., 2000. Macroscopic dielectric constant for microstructures of sedimentary rocks, Granular matter 2, Springer- Verlag, 137-141.

Howell, B. F., \& Licastro, P. H., 1961. Dielectric behavior of rocks and mineral, Am. Mineral., 46, 269-288.

Jonscher, A. K., 1983. Dielectric Relaxation in Solids, Chelsea Press , 219-226.

Jonscher, A. K., 1999. Dielectric relaxation in solids, J. Phys. D. Appl. Phys, 32, 57-70.

Kershaw, S., 2000. Quaternary reefs of Northeastern Sicily: Structure and growth controls in an unstable tectonic setting, Journal of Coastal Research, 16, 1037-1062.

Knight, R., 1983. The use of complex plane plots in studying the electrical response of rocks, J. Geomag. Geoelectr., 35, 767-776.

Knight, R.J., \& Nur, A., 1987. The dielectric constant of sandstones, $50 \mathrm{kHz}$ to $4 \mathrm{MHz}$, Geophysics, 52, $644-654$.

Lesmes, David, P., \& Dale, M.F., 2001. Dielectric spectroscopy of sedimentary rocks, Journal of Geophysical Research., 106, B7, 13329-13346.

Macdonald, J.R., (Ed). 1987. Impedance Spectroscopy, Wiley, New York.

Maxwell, J.C., 1891. A Treatise on Electricity and Magnetism 3rd edn, vol 1 (Oxford: Clarendon), reprinted 1954 (New York: Dover), p 246.

Nover, G., Heikamp, S., \& Freund, D., 2000. Electrical impedance spectroscopy used as a tool for the detection of fractures in rock samples exposed to either hydrostatic or triaxial pressure conditions, Natural Hazards, $21,317-330$.

Ruffet, C., Gueguen, Y., \& Darot M., 1991a. Rock conductivity and fractal nature of porosity, Terra Nova, 3, 265-275.

Ruffet, C., Gueguen, Y., \& Darot, M., 1991b. Complex measurements and fractal nature of porosity, Geophysics, 56, 758-768.

Ruffet, C., Gueguen, Y., \& Darot, M., 1991. Complex conductivity measurements and fractal nature of porosity, Geophysics, 56, 758-768.

Siddiqi, G., Evans, B., Dresen, G., \& Freund, D., 1997. Effect of semi brittle deformation on transport properties of calcite rocks. J. Geophys. Res., 102, (B7), 14765-14778.

Wagner, K.W., 1914. Erklärung der dielekrischen Nachwirkungsvorgänge auf Grund Maxwellscher Vorstellungen, Archiv für Electrotechnik II(9), 371-387, (in German). 